\section{It Is What It Is, but It Shouldn't Be: The Science of Ambiguity}

\author{
Thomas E. Marler ${ }^{1}$ and Olympia Terral \\ Western Pacific Tropical Research Center, College of Natural and Applied \\ Sciences, University of Guam, UOG Station, Mangilao, Guam 96923
}

Additional index words. education, graduate training, horticulture engagement, outreach, professional development

Pursuit of improved scientific writing demands that we illuminate ambiguous or misused words or phrases that frequently show up in the literature. Once identified, a concerted effort to diminish the incidence of misused phrases would help to clarify the accumulating literature. Toward that end, we have looked in various horticulture journals to determine whether there are clear examples of misused words that should be avoided. Graduate student advisors and journal editors may use their position to influence improved writing by decreasing the misuse of words and phrases.

\section{Beginning Sentences with the Word "It"}

Much has been written about the appropriateness of beginning a sentence with the word "it," and for most literary disciplines, the practice is tolerated as a writing style when the author deems necessary. However, in scientific writing, the focus is clarity and integrity of technical content, not promulgating style for the sake of technique.

As a pronoun, the word "it" requires an unambiguous antecedent for the reader to understand the sentence and continue reading without confusion. The antecedent should precede the pronoun and no additional subject should appear between the pronoun and its antecedent to maximize clarity. When a new thought is introduced into a manuscript by beginning a sentence with the vague pronoun "it," which has no meaning by itself, there is no antecedent preceding the pronoun. Therefore, the sentence begins with ambiguity and the reader is forced to proceed well into the sentence before finding the main subject buried therein.

To quantify the extent to which this habit is used by authors, we conducted a search of the phrase "it is" within every issue of HortScience in 2013 and every issue of HortTechnology and Journal of the American Society for Horticultural Science (JASHS) in 2012 and 2013 (12 issues per journal). For each time the phrase was identified at the beginning of a sentence, we analyzed the paragraph and preceding paragraph to determine if the pronoun was used ambiguously. If so, we considered

Received for publication 29 Apr. 2014. Accepted for publication 2 July 2014.

${ }^{1}$ To whom reprint requests should be addressed; e-mail tmarler@uguam.uog.edu. the sentence a case of misuse. Following are some of the filters we used in the process. Whenever the sentence began with an adverb such as "however," "additionally," "therefore," or "furthermore," and the misused "it" immediately followed the adverb, we added the occurrence to our tally. Whenever the sentence began with a conjunction such as "although," and the misused "it" immediately followed the conjunction, we also added the occurrence to our tally. Whenever the "it is" that was positioned at the beginning of a sentence referred to an antecedent positioned before the sentence, this was deemed an appropriate use of the pronoun. Interestingly, appropriate use of the phrase occurred most often within cultivar and germplasm description papers, in which the taxon being described served as the antecedent.

Whenever the "it is" was positioned within the sentence, we did not add the occurrence to our tally despite misuse of the pronoun. Our goal with this synthesis is to improve clarity within the accumulating horticulture literature, and these sentences usually contained the main topic early in the sentence. For example, a sentence beginning with "For appropriate placement of fertilizer, it is necessary to consider..." contains an incorrect use of "it" but the subject of the sentence is clearly conveyed before that mistake. Therefore, clarity was not compromised in these occurrences despite the misuse of the pronoun. As a result, our tally represents an underestimation of the total frequency of pronoun misuse. four to 25 times per issue for HortScience, one to 19 times per issue for $J A S H S$, and five to 26 times per issue for HortTechnology. We found no journal issue that was devoid of this mistake. As a means of standardizing the frequency of misuse, we computed the quotient defined by frequency of misuse divided by number of articles within each issue. We also searched numerous journals that horticulturists use for publishing and learned that every issue of these journals also contained numerous examples of this mistake. Misuse of the pronoun "it" at the beginning of a sentence occurred roughly one time for every two articles at the low end of the range for HortScience and two times per article at the upper end of the range for the Australian Journal of Agricultural and Resource
Inaccurate use of the pronoun "it" occurred
Economics (Table 1). Interestingly, all three ASHS journals were positioned at the low end of the range.

The most common initiation of sentences with this misused pronoun was "It is "something' that..." Within ASHS journals, some frequently used words for the "something" were "possible," "important," "likely," and "noteworthy." In many cases, authors positioned even more words before "that," creating a writing style that minimized clarity and buried the main subject farther into the sentence. For example, instead of "it is established that...," we might find "it is now quite well established by horticulturists that..." In other cases, authors amplified confusion by using the pronoun "it" two times in one sentence. For example, "it is uncertain whether it is derived directly from Asian ancestors or not." The second "it" in this example referred to the antecedent that was identified in the previous sentence such that the ambiguous pronoun that was devoid of an antecedent was positioned between the second pronoun and its antecedent.

The most compelling argument for paying specific attention to the use of "it" is to respect the many scientists who speak and write English as a foreign language. Using an active voice typically creates greater ease of comprehension for these readers. Beginning a sentence with an undefined pronoun, then burying the unambiguous primary subject far into the sentence, is a less economical writing style that adds unnecessary space filler.

\section{Description of Species as "New Species"}

The definition of "species" is heterogeneous, and species concepts are elusive in biology. However, the time needed for a species to emerge as a distinct taxon is lengthy regardless of one's inclination toward the traditional biological species concepts of reproductive isolation and separation in nature or toward the phylogenetic, cladistic, or morphological concepts. When a species is described, the taxon has not migrated from non-existent to new; it has migrated from undescribed to described. When a species becomes extinct before being described, it was not a taxon that failed to exist before extinction; it was a distinct species despite being undescribed. Therefore, claiming to describe a new species is unjustifiably anthropocentric. The taxon that acquired the human-imposed epithet within a scientific publication had been a distinct species long before the describing authority was born. Taxonomists keen on the prospect of making the claim of something new can use "newly recognized species" or "newly described species" or a species that is "new to science" or "new to academia" to fulfill that personal need.

\section{"Dark Adaptation" in Papers on Chlorophyll Fluorescence}

Adaptation of organisms entails adjustments or changes in the organisms that allow them to become more suited to thrive and 
Table 1. The quotient derived by dividing the number of articles in an issue into the number of times sentences are inaccurately begun with the pronoun 'it' within the same issue for representative journals that horticulturists use for publication.

\begin{tabular}{lr}
\hline Journal name & Quotient \\
\hline Agriculture, Ecosystems \& Environment & 1.62 \\
American Journal of Botan & 1.00 \\
Annals of Applied Biology & 1.40 \\
Annals of Botany & 1.82 \\
Australian Journal of Agricultural and Resource Economics & 2.00 \\
Botanical Journal of the Linnean Society & 0.94 \\
Crop and Pasture Science & 1.00 \\
Economic Botany & 1.11 \\
Fruits & 1.20 \\
Functional Plant Biology & 1.13 \\
Horticultural Science \& Biotechnology & 0.71 \\
HortScience & 0.50 \\
HortTechnology & 0.65 \\
Journal of Agriculture Economics & 1.71 \\
Journal of the American Society for Horticultural Science & 0.79 \\
New Phytologist & 1.21 \\
Oikos & 1.13 \\
Physiologia Plantarum & 1.09 \\
Plant, Cell \& Environment & 1.86 \\
Science & 0.89 \\
Soil Science & 0.56 \\
The Plant Journal & 1.47 \\
Weed Biology and Management & 0.86 \\
\hline
\end{tabular}

survive in their environment. Therefore, changing the light environment of a leaf for a few minutes within a horticultural experiment does not generate an adaptation of that leaf.

We performed a search within all ASHS journals for the phrase "chlorophyll fluorescence" to quantify how often this mistake occurred. We probed the connotations of the papers that described chlorophyll fluorescence measurement on intact organs that generated the variable $\mathrm{Fv} / \mathrm{Fm}$. We did not include papers describing fluorescence variables that were recorded without an imposed dark period or papers that cited methods in earlier publications.

Only $25 \%$ of the papers in ASHS journals used appropriate terminology for the methods that are used to produce darkened photosynthetic organs made ready for the excitation light from the instrumentation. Some of these authors described leaves that were kept in a darkened room or stated that a certain number of minutes of darkness was provided and described how the darkness was imposed. Alternatively, some authors said that manufacturers' cuvettes were used to provide the required darkness. In contrast to these examples of appropriate description of methods, $69 \%$ of the papers that reported $\mathrm{Fv} / \mathrm{Fm}$ in ASHS journals inappropriately used the term "dark-adapted" or "dark adaptation" to describe the few minutes of darkness supplied to the photosynthetic organs. The range in duration of darkness that we found in the various papers was 6 to 40 min. Authors of the remaining $6 \%$ of the papers failed to mention that darkness was required to measure $\mathrm{Fv} / \mathrm{Fm}$, thereby failing to pass the fundamental requirement of methods that can be repeated by other researchers.

Values of $\mathrm{Fv} / \mathrm{Fm}$ derived from darkened photosynthetic organs reflect the potential quantum efficiency of PSII and are viewed as a discerning indicator of photosynthetic performance. Portable, user-friendly, relatively inexpensive fluorometers are marketed by various manufacturers, and most of the manuals from these manufacturers describe the use of their cuvettes as "adapting" the leaves to darkness. This is likely the source of the frequent mistake when describing chlorophyll fluorescence experimental methods in ASHS journals. However, these manufacturers are in the business of selling instruments. Authors and editors are in the business of pursuing clarity and accuracy in the horticulture literature. Because no plant is capable of adapting to a change in light level within 6 to $40 \mathrm{~min}$, we suggest that authors begin to refrain from repeating the misuse of this word.

\section{Gas Exchange Variables Are Not Rates}

Water or carbon dioxide gas exchange data are frequently described by the terms photosynthetic rate, respiration rate, or transpiration rate. A rate is the quantity of something measured against another scale. These physiology variables represent a flux, not a rate. We queried the cumulative archives of ASHS journals to learn that "photosynthetic rate" was used in more than 2600 papers, "respiration rate" was used in more than 1900 papers, and "transpiration rate" was used in more than 2000 papers. We suggest the use of "net photosynthesis," "net $\mathrm{CO}_{2}$ assimilation," "respiration," or "transpiration" for these variables. If authors have a preference for continuing the use of the term "rate" when describing these gas exchange data, then accuracy could be achieved by stating the data are presented as "rate per unit area."

\section{Nitrogen-fixing Plant Species}

Plants that associate with nitrogen-fixing symbionts are not capable of converting $\mathrm{N}_{2}$ into $\mathrm{NH}_{3}$; they merely exploit the nitrogen that is fixed by their symbiont. The trait that sets them apart from other plants is their unique ability to enter into the intimate relationship with the actual nitrogen-fixing organism, yet these plants are routinely referred to as nitrogen-fixing plants in the horticulture literature, a term that is plainly inaccurate.

\section{Double Meaning of Statistical Terms}

The adjective "significant" and the noun "parameter" have fairly loose meanings in their generic use but narrow definitions within statistics. For statistics, we are taught that the term "significant" is used only when an accepted procedure is used to validate the probability that experimental results could not be explained by just chance alone. When writing horticultural manuscripts that include experimental results, using the term "significant" in the generic sense of stating something is of general importance is confusing because many readers understandably infer that the term refers to results from the statistical test that was described. For statistics, the term "parameter" refers to quantities derived from a population such as the mean and SD. Therefore, using "parameter" to define a measured or calculated response variable such as plant height is using the term in its generic sense, not as defined in statistics. Consider the example that a population of apple trees in receipt of a horticultural treatment exhibited a mean height of $310 \mathrm{~cm}$. If the author uses the generic meaning to refer to the variable plant height as a "parameter" within the manuscript, this writing style does not negate the fact that the statistical "parameter" is $310 \mathrm{~cm}$. The confusion is palpable because $310 \mathrm{~cm}$ is the parameter for the parameter, the unambiguous statistical sense for the former and the confusing generic sense for the latter. We suggest that the term "parameter" should be restricted to its statistical use in horticulture papers. Other suitable general synonyms are available for the generic "parameter" such as characteristic or response variable. In our example, $310 \mathrm{~cm}$ can be discussed as the parameter (mean) for the variable (plant height) without confusion.

\section{Error Handling}

The scholarly literature contains many examples of how to improve clarity in scientific writing (e.g., Denman, 2005; Lindsay, 2011; Oppenheimer, 2006; Strunk and White, 1979). Despite this abundance of literature devoted to improving writing style, these misused words and phrases appear institutionalized within horticulture journals rather than idiosyncratic. We suggest that the quality of accumulating scientific literature would greatly improve if authors would agree to migrate from misuse to disuse. We acknowledge that continued use of these words and phrases would not be debilitating to the horticulture profession, and opinions likely 
abound that this focus on semantics is trivial and unjustified in light of other agendas that are more vital. However, writing is a learned trait, not an innate trait, so these institutionalized habits were learned. This means discontinuation of the same habits can be learned. The frequency of misuse is considerable and in each example, the suggested change would increase accuracy with no possibility of decreasing clarity. Furthermore, a replacement word or phrase is easily identifiable in each of our examples.

Graduate student advisors and journal editors are ideally positioned to influence collective efforts of improved writing style. Development of skills in scientific communication is often ignored in graduate student training. If graduate advisors ensure these writing mistakes are not allowed in dissertations, the effort may release an emerging horticulturist whose writing style does not repeat such mistakes. Disparity in style of the review system of each journal's editorial team may explain the great disparity depicted in Table 1. We believe graduate advisors and journal editors could improve horticultural communication by becoming more disciplined in minimizing these prevalent writing mistakes.

"It" is in rhetorical humor that "it" is suggested that "it" should be stopped. In all seriousness, we suggest that use of the pronoun "it" should be restricted to cases in which an unambiguous antecedent is presented immediately before use of the pronoun. We contend that continued use of terms such as "new species," "dark adaptation," "photosynthetic rate," and "nitrogen-fixing plants" perpetuates misconceptions, especially for novice horticulturists. In the interest of accuracy and clarity, there are compelling reasons to find the collective will to discontinue these pervasive habits.

\section{Literature Cited}

Denman, T. 2005. How not to write: An office primer for the grammatically perplexed. Quirk Books, Philadelphia, PA.

Lindsay, D. 2011. Scientific writing = thinking in words. CSIRO Publishing, Collingwood, Australia.

Oppenheimer, D. 2006. Consequences of erudite vernacular utilized irrespective of necessity: Problems with using long words needlessly. Appl. Cogn. Psychol. 20:139-156.

Strunk, W., Jr. and E.B. White. 1979. The elements of style. 3rd Ed. MacMillan Publishing, New York, NY. 